\title{
MELHORAMENTO DO ALGODOEIRO NO ESTADO DE SÃO PAULO: OBTENÇÃO DA CULTIVAR IAC $21^{(1)}$
}

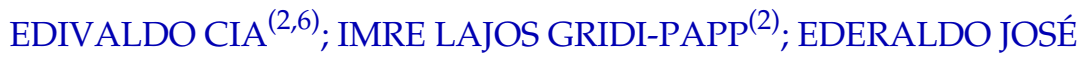 \\ CHIAVEGATO $^{(3,6)}$; NELSON PAULIERI SABINO ${ }^{(2,6)}$; JULIO ISAO KONDO ${ }^{(2)}$; \\ MARIA ANGÉLICA PIZZINATTO( ${ }^{(4,6)}$; NELSON BORTOLETTO ${ }^{(6)}$; LUIZ \\ HENRIQUE CARVALHO ${ }^{(2)}$ \\ RESUMO
}

\begin{abstract}
Neste trabalho é relatado o esquema utilizado para obtenção da cultivar IAC 21 realizado durante o período de 1981/82 a 1993/94. A cultivar foi originada por resseleção da IAC 19, direcionada principalmente para resistência a Colletotrichum gossypii var. cephalosporioides e nematóides, através do melhoramento genealógico. No início dos estudos as seleções e respectivas progênies foram comparadas à cultivar original IAC 19 e, posteriormente, a 'IAC 20'. Além das características econômicas de produção e qualidade da fibra, a linhagem foi avaliada para resistência múltipla às doenças: murchas de Fusarium e Verticillium, mancha-angular, ramulose e nematóides. A cultivar IAC 21 foi superior a 'IAC 19' em 6,2\%, em produtividade, na média geral dos experimentos, chegando a 18,1\% em solos altamente infectados por patógenos; $4,6 \%$ em produção de sementes, $2,4 \%$ em tenacidade da fibra e 6,9\% em tenacidade do fio, sem perdas significativas em outras características, com exceção da porcentagem de fibra que decresceu $2,6 \%$. Com respeito às doenças, apresentou melhores índices para murcha de Fusarium e ramulose mantendo-se, entretanto, no mesmo nível para as demais. Estudos preliminares mostraram que 'IAC 21' apresentou índices inferiores a 'CNPA ITA 90' para a anomalia "murchamento avermelhado". Devido às vantagens verificadas em relação a 'IAC 19' e 'IAC 20', 'IAC $21^{\prime}$ foi recomendada para plantio em todas as áreas do Estado de São Paulo, excluindo-se as de ocorrência do "murchamento avermelhado".
\end{abstract}

Palavras-chave: algodão, melhoramento, cultivar IAC 21, doenças.

\section{ABSTRACT \\ COTTON BREEDING IN THE STATE OF SÃO PAULO: DEVELOPMENT OF CULTIVAR IAC 21}

It is described the program to obtain cotton cultivar IAC 21 developed over the period of 1981/82 to 1993/94. The cultivar IAC 21 was originated from selections in 'IAC 19' population using the pedigree procedure taking into account some agronomical characteristics, and complemented with two specific breeding cycles for resistance to Colletotrichum gossypii var. cephalosporioides and to nematodes. Initially, comparisons of results were made to 'IAC 19', later to 'IAC 20'. Economical characteristics of production, fiber quality and multiple resistance to diseases (Fusarium wilt, Verticillium wilt, bacterial blight, ramulosis and nematodes) were evaluated. When compared to 'IAC

(1) Recebido para publicação em 9 de agosto de 2000 e aceito em 1 de fevereiro de 2001.

$\left({ }^{2}\right)$ Centro de Algodão e Fibrosas Diversas, Instituto Agronômico (IAC), Caixa Postal 28, 13001-970 Campinas (SP). E-mail: cia@cec.iac.br

( $\left.{ }^{3}\right)$ Departamento de Produção Vegetal, Escola Superior de Agricultura "Luiz de Queiroz", Universidade de São Paulo, Caixa Postal 9, 13418-900 Piracicaba (SP).

$\left({ }^{4}\right)$ Centro de Fitossanidade, IAC.

$\left({ }^{5}\right)$ Núcleo de Agronomia do Noroeste, Votuporanga, IAC.

(6) Com bolsa de produtividade em pesquisa do CNPq. 
$19^{\prime}$, the cultivar IAC 21 showed the following overall gains: $6.2 \%$ in yield, being of $18.1 \%$ on highly infected soil; $4.6 \%$ in seed yield; $2.4 \%$ in fiber tenacity and $6.9 \%$ in yarn tenacity, with no losses in other characters except to fiber that was lowered by $2.6 \%$ (about $1 \%$ in absolute value). There was an improvement in Fusarium wilt and ramulosis resistance, and no changes as to resistance to other diseases. A preliminary study revealed that the new anomaly called, in Brazil, "murchamento avermelhado" (reddish wilt or bronze wilt) affected 'IAC 21' more than the resistant cultivar CNPA ITA 90. By its advantages over 'IAC 19' and 'IAC 20', the cultivar IAC 21 was released in 1993, recommended for all the cotton areas of the State of São Paulo, except those where "murchamento avermelhado" occurred.

Key words: cotton, breeding, cultivar IAC 21, diseases.

\section{INTRODUÇÃO}

No período de 1975 a 1983, a cotonicultura paulista foi marcada por uma série de substituições de cultivares. Com o lançamento de 'IAC 17', em 1975, foi estabelecido um novo patamar de produtividade, cerca de 30\% superior ao das cultivares então em cultivo (Gridi-PAPP et al., 1984). Ganhos em qualidade da fibra e resistência à bactéria causadora da manchaangular (Xanthomonas axonopodis pv. malvacearum) foram obtidos com o lançamento da cultivar IAC 18 em 1977 (Gridi-PAPP et al., 1985). Em 1980 e 1983 foram lançadas, respectivamente, 'IAC 19' (GRIDI-PAPP et al., 1991) e 'IAC 20' (GRIDI-PAPP et al., 1993), proporcionando novos ganhos em produtividade, qualidade da fibra - especialmente com 'IAC 19' - e resistência a doenças e pragas (GRIDI-PAPP et al., 1991, 1993 e 1994). Essas duas cultivares foram utilizadas no Estado de São Paulo e em outros estados do Brasil na década de oitenta, permanecendo 'IAC 20' até 1996.

Nessa época buscava-se uma cultivar com a produtividade de 'IAC 20 ', a qualidade da fibra de 'IAC $19^{\prime}$ e um nível superior de resistência múltipla às doenças e pragas mais importantes na cotonicultura paulista. A linha de trabalho que oferecia maior probabilidade de avanço rápido nesse sentido compreendia a resseleção de 'IAC 19'. Dessa maneira visava-se, principalmente, elevar sua produtividade a níveis semelhantes ou superiores a 'IAC 20', e melhorar sua resistência a doenças e pragas, sobretudo à ramulose. Como a diferença média de produtividade entre as duas cultivares não era superior a $5 \%$, as perspectivas de atingir os objetivos mencionados, sem perdas sensíveis em qualidade da fibra, eram promissoras.

A execução deste trabalho, compreendendo as diversas fases de seleção e estudos, assim como os resultados dos ensaios regionais que permitiram a aprovação da linhagem IAC 19-809 para lançamento como nova cultivar, são relatados na presente pesquisa.

O objetivo deste estudo foi obter uma nova cultivar IAC através do esquema de melhoramento de seleção genealógica adotado no Centro de Algodão e Fibrosas Diversas do Instituto Agronômico.

\section{MATERIAL E MÉTODOS}

Foi adotado o esquema de seleção genealógica, no qual as seleções na cultivar IAC 19 foram realizadas, inicialmente, no ano agrícola de 1981/82, em Tietê (SP), no campo de produção de sementes; a seguir, nos anos agrícolas de 1983/84 e 1984/85 em Piracicaba (SP), em testes para resistência à ramulose e, em 1986/87 e 1987/88, em Ituverava (SP) e Tatuí (SP) em testes para resistência a nematóides. O quadro 1 resume a evolução dos trabalhos a partir das seleções iniciais e respectivos testes para doenças.

Os testes de avaliação dos materiais genéticos foram conduzidos, também anualmente, em áreas de infestação natural mantidas pelo Programa de Algodão, em Estações Experimentais do IAC e fazendas particulares, com exceção da bactéria Xanthomonas axonopodis pv. malvacearum Dye (mancha-angular) e do fungo Colletotrichum gossypii South. var. cephalosporioides (ramulose), os quais foram inoculados em campo, no Núcleo Experimental de Campinas e na Estação Experimental de Agronomia de Piracicaba respectivamente. A bactéria foi inoculada em plantas com 70 dias de idade, aproximadamente, e o fungo da ramulose, em plantas com 15 e 30 dias de idade (CIA et al., 1975; CiA et al., 1982). Os demais agentes estudados foram: Fusarium oxysporum f. vasinfectum (murcha de Fusarium), Verticillium dahliae (murcha de Verticillium) e nematóides (Meloidogyne incognita, Rotylenchulus reniformis, Pratylenchus brachyurus e Helicotylenchus sp.).

Os testes para avaliação das doenças compreenderam parcelas de uma linha, com 4 ou $5 \mathrm{~m}$ de comprimento, repetidas de duas a quatro vezes, conforme as áreas infectadas e sementes disponíveis. A cada seis ou oito parcelas foram intercaladas uma linha de testemunha resistente e uma de testemunha suscetível.

A avaliação do nível de resistência a esses patógenos baseou-se em notas e índices de resistência descritos por GRIDI-PAPP et al. (1994). Estabeleceramse, também, índices de tolerância para os dois fungos causadores de murcha, através do quociente do número de plantas que produziram três ou mais 
Quadro 1. Origem de 'IAC 21': evolução genealógica de linhagens de 'IAC 19' nos anos agrícolas de 1981/82 a 1993/94

\begin{tabular}{|c|c|c|}
\hline & Ensaio/Teste & Evolução \\
\hline $81 / 82$ & $\begin{array}{c}\text { Campo de aumento (IAC } \\
\text { 19) E.E.Tietê }\end{array}$ & $\begin{array}{c}\text { Seleção individual } \\
\text { n. }{ }^{\circ} 1129 \\
\text { (entre } 579 \text { plantas) }\end{array}$ \\
\hline $82 / 83$ & Progênie IAC 19 & Trat. $^{\circ} 16$ (19-241) \\
\hline $83 / 84$ & $\begin{array}{l}\text { Ensaio novas linhagens e } \\
\text { teste anual ramulose }\end{array}$ & $\begin{array}{c}\text { Seleção individual } \\
\text { n. }{ }^{.} 3383\end{array}$ \\
\hline $84 / 85$ & $\begin{array}{l}\text { Teste progênies de } \\
\text { seleção individual } \\
\text { ramulose }\end{array}$ & $\begin{array}{l}\text { Seleção massal } \\
\text { n. }^{\circ} 3289\end{array}$ \\
\hline $85 / 86$ & Progênie IAC 19 & Linhagem IAC 19-521 \\
\hline $86 / 87$ & $\begin{array}{l}\text { Ensaio novas linhagens e } \\
\text { teste anual nematóides }\end{array}$ & $\begin{array}{l}\text { Seleção individual } \\
\text { n. }{ }^{\circ} 3146\end{array}$ \\
\hline $87 / 88$ & $\begin{array}{l}\text { Teste progênies seleção } \\
\text { individual (Nematóides) }\end{array}$ & $\begin{array}{l}\text { Seleção massal } \\
\text { n. }^{\circ} 3157\end{array}$ \\
\hline $88 / 89$ & $\begin{array}{l}\text { Ensaio progênies } \\
\text { IAC } 19\end{array}$ & Linhagem IAC 19-809 \\
\hline $89 / 90$ & $\begin{array}{l}\text { Ensaio nova linhagem } \\
\text { IAC } 19\end{array}$ & Linhagem IAC 19-809 \\
\hline $90 / 91$ & $\begin{array}{l}\text { Ensaio regional } \\
\text { variedades }\end{array}$ & Linhagem IAC 19-809 \\
\hline $91 / 92$ & $\begin{array}{l}\text { Ensaio regional } \\
\text { variedades }\end{array}$ & Linhagem IAC 19-809 \\
\hline $92 / 93$ & $\begin{array}{l}\text { Ensaio regional } \\
\text { variedades }\end{array}$ & Linhagem IAC 19-809 \\
\hline 93/94 & $\begin{array}{l}\text { Ensaio regional } \\
\text { variedades }\end{array}$ & Cultivar IAC 21 \\
\hline
\end{tabular}

capulhos pelo número de plantas deixadas no desbaste (estande inicial), conforme CiA et al. (1978). Tal procedimento foi adotado devido à ocorrência de correlação negativa entre resistência e produtividade, e a seleção de plantas, somente pelo índice de resistência, poderia resultar em linhagens pouco produtivas.

Como as notas e os índices empregados variaram em sentido inverso, de suscetível a resistente, foram efetuadas as transformações sugeridas por GRIDI-PAPP et al. (1982), obtendo-se, para todos os fatores adversos, índices uniformizados que variaram numericamente de 0 (morte) a 1 (imunidade).

Visando diminuir o efeito da ocorrência irregular das doenças e nematóides nas áreas dos testes, computaram-se índices relativos das parcelas experimentais, dividindo-se o índice obtido para cada uma pela média dos índices das duas testemunhas resistentes mais próximas.

Finalmente, foram calculadas as médias dos índices relativos assim computados, para cada li- nhagem e cada patógeno, e por meio da média geométrica desses valores foi calculado um índice múltiplo de resistência às várias doenças. Esse índice múltiplo foi padronizado, dividindo-se por $(1+\mathrm{s})$, considerando que s representa o desvio-padrão das médias dos índices relativos a determinada linhagem (GRIDI-PAPP et al., 1994). O índice de resistência múltipla assim obtido permitiu a avaliação global das linhagens em estudo. Considerou-se, também, para avaliação global dos resultados, um índice de segurança, definido como o produto do índice múltiplo não-padronizado, pelo menor índice observado entre os patógenos avaliados para determinada linhagem.

A seleção em testes de resistência obedeceu a um ciclo de dois anos, tendo sido estudadas, no primeiro ano as progênies das plantas selecionadas para determinado patógeno, retornando-se, no ano seguinte, aos testes para confirmação da sua resistência e a realização de seleção massal (GRIDI-PAPP et al., 1994).

Os estudos das demais características econômicas de cada material selecionado foram realizados, paralelamente, em ensaios de progênies, de linhagens e ensaios regionais, localizados, nos primeiros anos, no Núcleo Experimental de Campinas e posteriormente em várias regiões produtoras do Estado de São Paulo. Esses ensaios tiveram delineamentos próprios, de acordo com esquema de seleção genealógica adotado pelos melhoristas do Centro de Algodão e Fibrosas Diversas do Instituto Agronômico, (CAVALERI e GRIDI-PAPP, 1993). Foram avaliadas a produção, massa de um capulho, massa de 100 sementes, porcentagem de fibra, altura de plantas e precocidade. A fibra foi analisada, conforme o ano, em aparelhos convencionais ou no equipamento SPINLAB 900 SYSTEM (HVI), variando, portanto, os valores das características estabelecidas. Foram determinadas, ainda, a tenacidade e elongação do fio num conjunto de microfiação. A determinação das características de fibra e produção foi efetuada segundo apresentação de GRIDI-PAPP et al. (1993). Paralelamente a esses ensaios, foi estudado o comportamento dos materiais em presença dos agentes causadores das murchas de Fusarium e de Verticillium, ramulose, mancha- angular e nematóides.

\section{RESULTADOS E DISCUSSÃO}

No início dos trabalhos foram selecionadas 579 plantas, e, nas melhores progênies, foram feitas seleções no ano seguinte. A progênie IAC 19-241 (planta n. ${ }^{\circ} 1129$ ) apresentou bom desempenho no campo e passou a ser estudada no ano agrícola de 1983/84 nos Ensaios de Novas Linhagens de 'IAC 19'. A evolução dos trabalhos pode ser observada no quadro 1 . As 
características agronômicas e tecnológicas dessa linhagem constam do quadro 2. Pode-se observar que apresentou, em geral, níveis economicamente viáveis, superando a cultivar IAC 19, principalmente em tenacidade da fibra, avaliada pelo índice Pressley $1 / 8^{\prime \prime}\left(\right.$ g.tex $\left.{ }^{-1}\right)$ e resistência intrínseca $\left(\right.$ g.tex $\left.{ }^{-1}\right)$. Paralelamente, nesse mesmo ano, tal linhagem foi estudada nos testes de resistência às doenças consideradas neste estudo. Podem-se observar os resultados no quadro 2, onde a linhagem apresenta, em geral, comportamento superior ao de 'IAC 19', com ênfase na elevada resistência às murchas de Fusarium e de Verticillium. Particularmente animadora foi a melhoria verificada no nível de resistência à ramulose, deficiência já observada em 'IAC 19'.

Nos testes de resistência a doenças, especificamente para ramulose, outras seleções individuais foram realizadas, das quais uma nova planta se destacou (planta n. ${ }^{\circ} 3383$ da linhagem 19-241). A progênie dessa planta voltou a ser testada no ano agrícola de 1984/85, em presença de ramulose, dentro do ciclo bianual de seleção, repetindo o bom desem- penho e fornecendo a seleção massal n. ${ }^{\circ} 3289$, cuja descendência recebeu, após o estudo de progênie, a denominação 'IAC 19-521'. Como linhagem eleita, foi avaliada no ano agrícola de 1986/87 no Ensaio de Novas Linhagens de IAC 19, bem como em testes para resistência a nematóides, tendo em vista o seu fraco desempenho verificado nas avaliações anteriores. $\mathrm{O}$ desempenho dessa linhagem comparado ao de 'IAC $19^{\prime}$ é também apresentado no quadro 2 .

Nota-se que a linhagem foi mais produtiva que a cultivar original, mantendo a superioridade para tenacidade da fibra (Pressley $1 / 8$ " e resistência intrínseca), uniformidade do comprimento da fibra e massa de 100 sementes. Quanto às demais características, os resultados foram estatisticamente semelhantes. Do mesmo modo, com respeito às doenças, confirmou a superioridade de resistência para murchas de Fusarium e de Verticillium e evoluiu em relação à resistência à ramulose, evidentemente em face da pressão de seleção exercida em presença do patógeno, nos dois últimos anos de testes. Observa-se, porém, que seu desempenho para resistência à mancha-angular e a

Quadro 2. Dados extraídos dos Ensaios de Competição de Novas Linhagens de 'IAC 19', (delineamento em quadrado latino com seis tratamentos) obtidos em três ciclos de seleção e nos testes de resistência a doenças nos anos de 1983/84, 1986/87 e 1989/90

\begin{tabular}{|c|c|c|c|c|c|c|}
\hline \multirow[t]{2}{*}{ Características } & \multicolumn{2}{|c|}{$1983 / 84$} & \multicolumn{2}{|c|}{$86 / 87$} & \multicolumn{2}{|c|}{$89 / 90$} \\
\hline & IAC 19 & IAC 19-241 & IAC 19 & IAC 19-521 & IAC 19 & IAC $19-809$ \\
\hline Produção (kg.parcela ${ }^{-1}$ ) & - & - & $1,64 \mathrm{ab}$ & 1,86 a & $1,18 \mathrm{~b}$ & $1,31 \mathrm{ab}$ \\
\hline Fibra (\%) & $38,0 a^{(1)}$ & 37,9 a & 37,6 a & $38,0 \mathrm{a}$ & $37,8 \mathrm{ab}$ & $37,0 \mathrm{~b}$ \\
\hline Massa de 100 sementes (g) & $14,2 \mathrm{a}$ & $14,1 \mathrm{a}$ & $14,2 \mathrm{bc}$ & $14,7 \mathrm{a}$ & $13,8 \mathrm{~b}$ & $15,0 \mathrm{a}$ \\
\hline Massa de 1 capulho(g) & $7,4 \mathrm{ab}$ & $7,4 \mathrm{ab}$ & $7,1 \mathrm{a}$ & $7,5 \mathrm{a}$ & $6,5 \mathrm{a}$ & $6,9 \mathrm{a}$ \\
\hline Precocidade (\%) de produção & - & - & $7,0 \mathrm{ab}$ & $7,1 \mathrm{a}$ & 7,8 a & $6,6 \mathrm{~b}$ \\
\hline Comprimento $(\mathrm{mm})$ de fibra & $28,0 \mathrm{ab}$ & $28,2 \mathrm{ab}$ & $28,4 \mathrm{ab}$ & $27,8 \mathrm{bc}$ & $28,3 \mathrm{~b}$ & $29,3 \mathrm{a}$ \\
\hline Uniformidade do comprimento $2,5 \%$ & $45,5 \mathrm{ab}$ & 46,2 a & $46,1 \mathrm{~d}$ & $49,5 \mathrm{a}$ & $44,9 \mathrm{~b}$ & $46,5 \mathrm{a}$ \\
\hline Micronaire (índice) & $5,1 \mathrm{a}$ & $5,1 \mathrm{a}$ & $5,1 \mathrm{~b}$ & 5,4 a & $5,1 \mathrm{ab}$ & $5,0 \mathrm{~b}$ \\
\hline Pressley 1/8" $\left(\right.$ g.tex $\left.^{-1}\right)$ & $23,2 \mathrm{~b}$ & 24,5 a & $23,3 \mathrm{~b}$ & $23,8 \mathrm{ab}$ & $23,2 \mathrm{bc}$ & 24,1 a \\
\hline Resist. intrínseca (g.tex-1) & $22,2 \mathrm{a}$ & $23,4 \mathrm{a}$ & $23,7 \mathrm{~b}$ & $25,3 \mathrm{a}$ & $21,3 \mathrm{~b}$ & $22,1 \mathrm{ab}$ \\
\hline Maturidade (\%) & $57,5 \mathrm{a}$ & $58,0 \mathrm{a}$ & $64,1 \mathrm{~b}$ & $66,3 \mathrm{ab}$ & $55,1 \mathrm{~b}$ & $57,9 \mathrm{ab}$ \\
\hline Resist. fio (RKM) & $14,7 \mathrm{~b}$ & $15,3 \mathrm{ab}$ & $14,5 \mathrm{~b}$ & $14,7 \mathrm{ab}$ & $15,1 \mathrm{~b}$ & $16,7 \mathrm{a}$ \\
\hline \multirow[t]{2}{*}{ Elongação fio (\%) } & $6,4 \mathrm{a}$ & $6,2 \mathrm{a}$ & $6,5 \mathrm{~b}$ & $6,9 \mathrm{ab}$ & $6,8 \mathrm{a}$ & $6,9 \mathrm{a}$ \\
\hline & \multicolumn{6}{|c|}{ Índices relativos } \\
\hline Mancha-angular-Nota & - & - & 0,944 & 0,625 & 0,733 & 0,902 \\
\hline Murcha Fusarium-Tol.\% & 0,540 & 0,626 & 0,704 & 0,752 & 1,118 & 1,201 \\
\hline Murcha Fusarium-Res.\% & 0,663 & 1,129 & 0,639 & 1,307 & 0,532 & 1,532 \\
\hline Murcha Verticillium - Tol.\% & 0,768 & 0,884 & - & - & 0,747 & 0,678 \\
\hline Murcha Verticillium - Resist. \% & 0,530 & 1,602 & 1,289 & 1,475 & 1,300 & 0,774 \\
\hline Nematóides- Nota & 0,802 & 0,745 & 0,640 & 0,598 & 0,871 & 0,729 \\
\hline Ramulose - Nota & 0,543 & 0,617 & 0,515 & 0,699 & 0,531 & 0,764 \\
\hline
\end{tabular}

${ }^{(1)}$ Teste de Duncan a 5\%. 
nematóides, permaneceu relativamente inferior ao da cultivar original.

Considerando-se a importância desses dois fatores, principalmente os nematóides - limitantes para a cultura algodoeira -, intensificaram-se os trabalhos de seleção em presença desses dois patógenos. Dessa forma, procurou-se garantir, no mínimo, o comportamento da cultivar original, considerado até então de bom nível, além de estabilizar os ganhos já obtidos nas outras características.

No ano de 1986/87, paralelamente aos estudos de linhagens, a linhagem IAC 19-521 foi resselecionada no teste anual para nematóides entre linhagens de 'IAC 19'. A melhor planta (SI 3146), cuja progênie também se destacou na presença do parasita no ano seguinte, proporcionou ainda uma seleção massal (SM 3157) no teste para nematóides. A progênie dessa planta e a respectiva linhagem, denominada IAC 19809, foram eleitas no ano agrícola de 1987 / 88 para o Ensaio de Novas Linhagens. Os resultados da linhagem no ano agrícola de 1987/88 constam igualmente no quadro 2 .

A linhagem IAC 19-809 mostrou-se superior a 'IAC 19' em algumas características de grande importância econômica para o setor algodoeiro. A produtividade foi maior em cerca de $10 \%$, a uniformidade de comprimento da fibra e a tenacidade da fibra e do fio mantiveram-se em níveis mais elevados. Nas demais características agronômicas e tecnológicas de laboratório, apresentou comportamento semelhante a 'IAC 19'. Com relação ao conjunto dos índices de resistência às doenças, mostrou-se economicamente mais interessante e manteve-se superior, em relação à resistência para mancha-angular e nematóides, comparativamente às linhagens anteriores. Destacou-se, em particular, para maior resistência à ramulose, comportamento que 'IAC 19' deixava a desejar.

Essa linhagem foi eleita para integrar o Ensaio Regional de Variedades Paulistas a partir do ano agrícola de 1990/91. No quadro 3 são apresentados os dados médios de dois anos que comparam a linhagem IAC $19-809$ e a cultivar IAC 19, obtidos nos ensaios conduzidos regionalmente em 37 locais (18 locais em 1990/91 e 19 locais em 1991/92), distribuídos no Estado de São Paulo.

Avaliada regionalmente, a linhagem IAC 19-809 apresentou, em relação a 'IAC 19', melhoria de 6,2\% na produção média de algodão em caroço, $18,1 \%$ na produtividade dos ensaios em solos altamente infectados pelos patógenos, $4,6 \%$ na massa de sementes, $2,4 \%$ em tenacidade da fibra e $6,9 \%$ na tenacidade do fio, sem perdas significativas nas outras característi- cas, com exceção para porcentagem de fibra, que diminuiu em $2,6 \%$.

Os estudos desses dados e das avaliações anteriores e os relacionados à resistência a patógenos, a serem apresentados posteriormente, justificaram o lançamento da linhagem IAC 19-809 como cultivar IAC 21, visando o aumento da produção de sementes a partir do ano agrícola de 1992/93, e distribuídas comercialmente aos produtores no ano agrícola seguinte.

Não havendo mais interesse de cultivo entre os produtores do Estado de São Paulo pela cultivar IAC 19 , esta foi retirada do ensaio regional a partir do ano agrícola de 1992/93. Desse modo, a nova cultivar IAC 21 passou a ser comparada, nos anos de 1992/93, 1993/94 e 1994/95, com 'IAC 20', então em cultivo por todo o Estado. Os resultados médios comparativos são apresentados no quadro 4.

Como pode ser observado nesse mesmo quadro, 'IAC 21', comparada a 'IAC 20', apresentou, no período, melhoria de 1,9\% na produção de algodão em caroço, na média de 59 ensaios, e 18,3\% na produção dos ensaios em áreas comprovadamente com problemas de murcha de Fusarium, ramulose e nematóides. Mostrou-se também superior em 4,5\% na massa de 100 sementes, 2,2\% no comprimento de fibra, $5,8 \%$ na tenacidade da fibra e $4,7 \%$ na tenacidade do fio, sem perdas significativas nas demais características, com exceção para a porcentagem de fibra, a qual foi reduzida em $0,5 \%$. Resultados semelhantes foram obtidos em outros estados, como os observados por Almeida et al. (1995), ArANTES e Freire (1995), Lamas e Ferraz (1993) e Medeiros (1995).

Paralelamente a esses estudos, foram conduzidos os testes anuais para resistência aos principais patógenos e nematóides que ocorrem no Estado de São Paulo, cujos índices obtidos para 'IAC 19', 'IAC $20^{\prime}$ e 'IAC 21' encontram-se no quadro 5 . Os testes para os patógenos foram realizados individualmente, em condições de campo e com infestações naturais, com exceção da mancha-angular e da ramulose, para as quais as plantas foram inoculadas artificialmente. Estudos semelhantes, com resultados individuais para cada patógeno, foram realizados inicialmente nos EUA, em 1966 (BIRD, 1966). Os dados são apresentados em índices relativos às testemunhas de referência, conforme CIA et al. (1982).

Conforme já verificado nos testes realizados nos anos anteriores, a cultivar IAC 21 confirmou sua superioridade em relação a 'IAC 19', nos anos de 1989/90 e 1990/91, e a 'IAC 20', nos anos de 1989/90 a 1994/95. Apresentou melhores índices para murcha de Fusarium e ramulose, mantendo-se, entretanto, no mesmo nível para os demais fatores. 


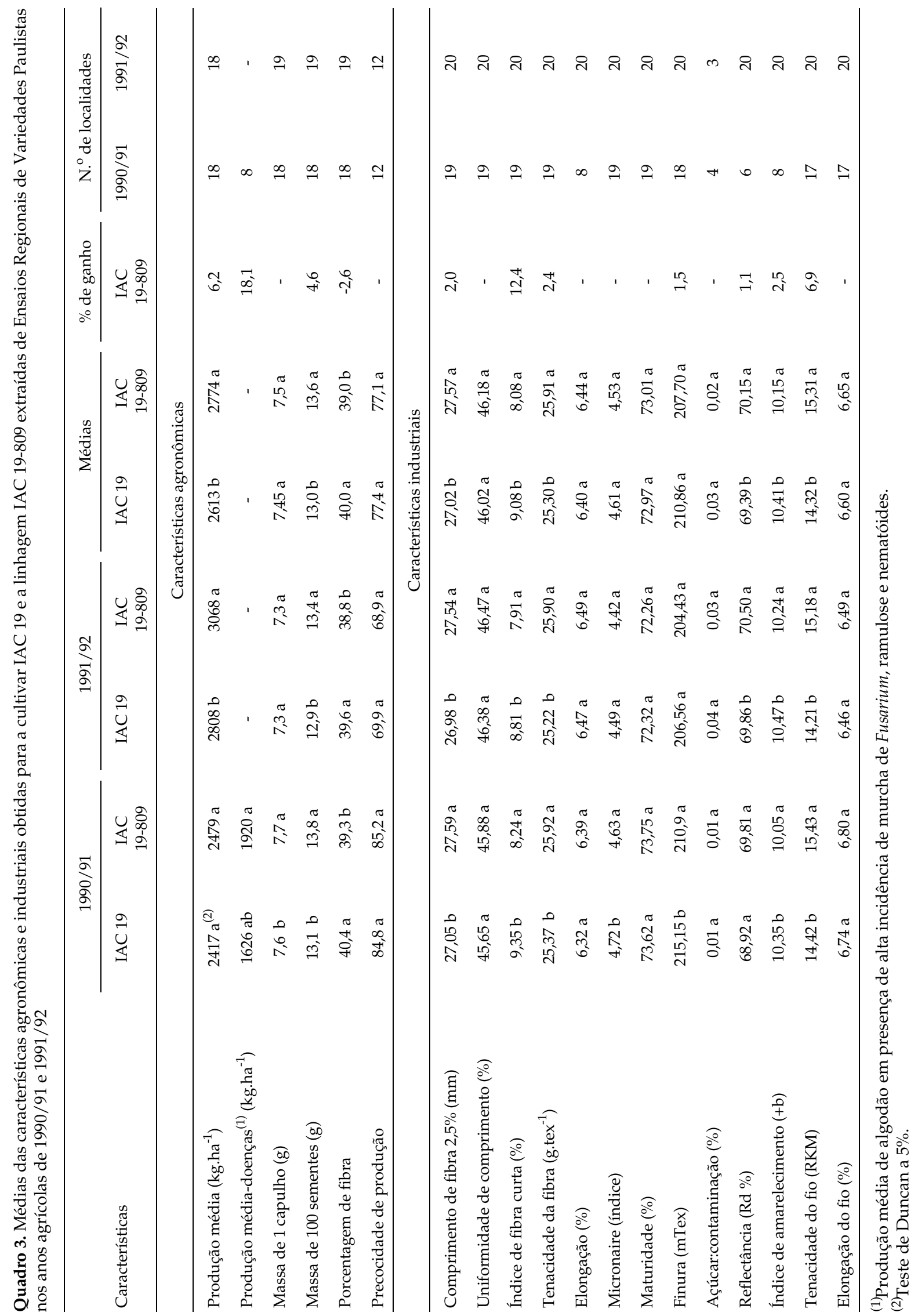


Melhoramento do Algodoeiro no Estado de São Paulo

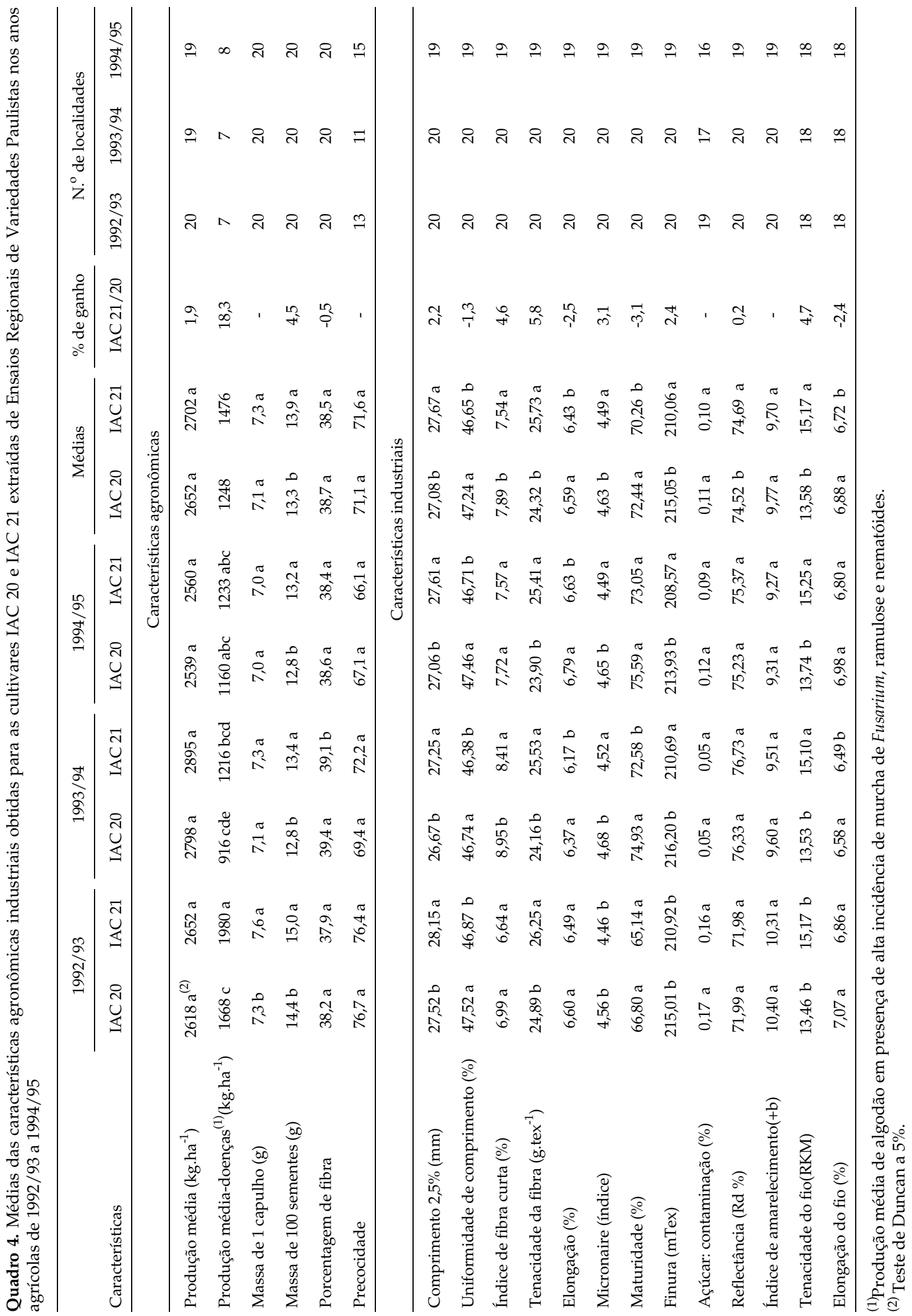


Quadro 5. Médias dos índices relativos, múltiplos e de segurança, obtidos para as cultivares IAC 19 (89/90 e 90/91), IAC 20 e IAC 21 (89/90 a 94/95) no estudo de resistência à mancha-angular, murchas de Fusarium e Verticillium, nematóides e ramulose

\begin{tabular}{|c|c|c|c|c|}
\hline \multirow{3}{*}{ Variáveis } & \multicolumn{4}{|c|}{ Anos agrícolas } \\
\hline & \multicolumn{2}{|c|}{$1989 / 90$ e $1990 / 91$} & \multicolumn{2}{|c|}{ 1989/90 a 1994/95 } \\
\hline & IAC 19 & IAC 21 & IAC 20 & IAC 21 \\
\hline Mancha-angular & 0,625 & 0,782 & 0,711 & 0,733 \\
\hline Murcha de Fusarium - tolerância & 0,886 & 0,962 & 0,860 & 0,918 \\
\hline Murcha de Fusarium - resistência & 0,712 & 1,315 & 0,883 & 1,354 \\
\hline Murcha de Verticillium - tolerância & 0,903 & 0,853 & $0,930^{(1)}$ & $0,853^{(1)}$ \\
\hline Murcha de Verticillium - tesistência & 1,142 & 0,921 & 0,914 & 0,844 \\
\hline Nematóides & 0,806 & 0,767 & 0,838 & 0,878 \\
\hline Ramulose & 0,539 & 0,915 & 0,704 & 0,958 \\
\hline Índice múltiplo & 0,739 & 0,922 & 0,805 & 0,932 \\
\hline Índice de segurança & 0,398 & 0,707 & 0,567 & 0,683 \\
\hline Índice resist. múltipla (padronizado) & 0,599 & 0,755 & 0,734 & 0,753 \\
\hline
\end{tabular}

(1)Médias de dois anos agrícolas: 1989/90 e 1990/91.

Na avaliação global dos resultados, pelos índices múltiplos, pode-se verificar a superioridade de 'IAC $21^{\prime}$ em relação às outras duas cultivares, indicando melhor comportamento frente aos patógenos considerados. Para esta análise, foi também incluído um índice que reflete o máximo de segurança aos patógenos estudados para cada material. Muitas vezes, dependendo do material, pode-se obter um índice múltiplo relativamente alto e um índice de segurança relativamente baixo. Pelos resultados expostos no quadro 5, observa-se maior variação entre os materiais para o índice de segurança em comparação aos outros dois índices múltiplos, demonstrando, com isso, que o primeiro deve também ser considerado na eleição dos materiais.

No presente estudo a cultivar IAC 21 apresentou bom índice de segurança, confirmado por FuzATTO et al. (1994), os quais demonstraram que 'IAC 21' foi melhor e mais estável em presença dos patógenos causadores da murcha de Fusarium, ramulose e de nematóides.

No mesmo ano de lançamento de 'IAC 21', foi detectada a ocorrência de uma anomalia causadora de prejuízos em algodoais, principalmente nas regiões de Leme e no oeste do Estado de São Paulo, bem como em outros estados. Essa anomalia, desconhecida até então (dezembro de 2000), foi denominada "murchamento avermelhado" (CHIAVEGATO et al. 1994). Estudos preliminares realizados em 1993/94 mostraram que essa cultivar comportava-se de maneira semelhante a 'IAC 20', em face do referido murchamento. Essas duas cultivares acusaram índices de resistência
Quadro 6. Índices relativos de "murchamento avermelhado" obtidos em quatro ensaios do melhoramento genético para as cultivares IAC 20, IAC 21 e CNPA ITA 90, no ano agrícola de 1993/94

\begin{tabular}{lccccc}
\hline \multirow{2}{*}{ Variedade } & \multicolumn{3}{c}{ Índices relativos ${ }^{(1)}$} & \multirow{2}{*}{ Médias } \\
\cline { 2 - 5 } & \multicolumn{2}{c}{ Teste anual } & \multicolumn{2}{c}{ Teste VNR } & \\
\cline { 2 - 5 } & Jaú & $\begin{array}{c}\text { Votupo- } \\
\text { ranga }\end{array}$ & Jaú & $\begin{array}{c}\text { Eldorado } \\
\text {-2-GO }\end{array}$ & \\
\hline IAC 20 & 0.585 & 0.824 & 0.816 & 0.934 & 0.790 \\
IAC 21 & 0.708 & 0.833 & 0.816 & 0.792 & 0.787 \\
CNPA ITA 90 & 1.150 & 1.050 & 1.023 & 1.250 & 1.118 \\
\hline
\end{tabular}

${ }^{(1)}$ Os índices relativos foram obtidos de avaliações, mediante incidência natural, nos ensaios de testes anuais e outros, para resistência a doenças, utilizando como testemunha a linhagem IAC 87/533.

inferiores ao de 'CNPA ITA 90', como pode ser observado no quadro 6.

\section{CONCLUSÃO}

Devido aos resultados alcançados pela cultivar IAC 21 em relação a 'IAC 19' e 'IAC 20', para as características econômicas de produção, qualidade de fibra e resistência múltipla a doenças, decidiu-se pelo seu lançamento, sendo recomendada em todas as áreas do Estado de São Paulo, excluindo-se as de ocorrência do "murchamento avermelhado". 


\section{REFERÊNCIAS BIBLIOGRÁFICAS}

ALMEIDA, W.P.; PIRES, J.R.; YAMAOKA, R.S.; TURKIEWICZ, L.; COSTA, A. Resultados do ensaio regional de variedades paranaenses no Estado do Paraná, em 1993/94 In: REUNIÃO NACIONAL DO ALGODÃO, 8., 1995, Londrina. Resumos... Londrina: EMBRAPA/CNPA, 1995. p.1.

ARANTES, E.M; FREIRE, E.C. Resultados dos ensaios regionais paulistas conduzidos no Mato Grosso 1993/94. In: REUNIÃO NACIONAL DO ALGODÃO, 8., 1995, Londrina. Resumos... Londrina: EMBRAPA/CNPA, 1995. p.32.

BIRD, L.S. Interrelation of resistance and escape of cotton from five major disease. Proceedings of Annual Cotton Disease Council, Memphis, v.26, p.92-1087, 1966.

CAVALERI, P.A.; GRIDI-PAPP, I.L. Algodão. In: FURLANI, A.M.C.; VIÉGAS, G.P. (Eds.).O Melhoramento de Plantas no Instituto Agronômico. Campinas, Instituto Agronômico, 1993. p.13-27.

CHIAVEGATO, E.J.; FUZATTO, M.G.; CIA, E.; PETTINELLI JÚNIOR, A. Avaliação preliminar de perdas na produção devidas à nova anormalidade do algodoeiro no Brasil. Bragantia, Campinas, v. 53, n.2, p.225258, 1994.

CIA, E.; FERRAZ, C.A.M.; GRIDI-PAPP, I.L.; SOAVE, J. Melhoramento do algodoeiro Gossypium hirsutum L. para resistência a Xanthomonas malvacearum (E.F.Smith) Dowson. Summa Phytopathologica, Piracicaba, v.1, p.177-186, 1975.

CIA, E.; FUZATTO, M.G.; GRIDI-PAPP, I.L.; SOAVE, J.; CIONE, J. Avaliação da incidência de ramulose do algodoeiro através de inoculação artificial. In: REUNIÃO NACIONAL DO ALGODÃO 2., 1982. Salvador. Resumos... Campina Grande: EMBRAPA/CNPA, 1982. p.241.

CIA, E.; GRIDI-PAPP, I.L.; FERRAZ, C.A.M. Correlações entre respostas de resistência apresentada por linhagens de algodoeiro (Gossypium hirsutum) a três agentes patogênicos. Summa Phytopathologica, Piracicaba, v.4, p.111-121, 1978.

FUZATTO, M.G.; CIA, E.; CHIAVEGATO, E.J. Estabilidade da produção de genótipos de algodoeiro em face da ocorrência de doenças enematóides. Bragantia, Campinas, v.53, n.1, p.47-52, 1994.

GRIDI-PAPP, I.L.; CIA, E.; FUZATTO, M.G. Uniformização de índices de avaliação em programas de melhoramento para resistência múltipla a fatores adversos. In: REUNIÃO NACIONAL DO ALGODÃO 2., 1982, Sal- vador. Resumos... Campina Grande: EMBRAPA/ CNPA, 1982. p.236.

GRIDI-PAPP, I.L.; CIA, E.; FUZATTO, M.G.; CAVALERI, P.A.; CHIAVEGATO, E.J.; FERRAZ, C.A.M.;SABINO, N.P.; KONDO, J.I.; SOAVE, J.; BORTOLETTO, N. Melhoramento do algodoeiro no Estado de São Paulo: obtenção da variedade IAC 18. Bragantia, Campinas, v.44, n.2, p.645-658, 1985.

GRIDI-PAPP, I.L.; CIA， E， FUZATTO, M.G.; CHIAVEGATO, E.J.; DUDIENAS, C.; PIZZINATTO, M.A; SABINO, J.C.; CAMARGO, A.P.; CAMPANA, M.P. Melhoramento do algodoeiro para resistência múltipla a doenças, nematóides e broca-da-raíz em condições de campo. Bragantia, Campinas, v.53, n.1, p.33-45, 1994.

GRIDI-PAPP, I.L.; CIA, E.; FUZATTO, M.G.; CAVALERI, P.A.;CHIAVEGATO, E.J.;SILVA, N.M.;CARVALHO, L.H.; SABINO, N.P.; KONDO, J.I.; SUGIMORI, M. H.; SOAVE, J.; FERRAZ, C.A.M. Melhoramento do algodoeiro no Estado de São Paulo: obtenção da variedade IAC 19. Bragantia, Campinas, v.50, n.2, p.181-194, 1991.

GRIDI-PAPP, I.L.;FUZATTO, M.G.;CAVALERI,P.A.;CIA, E.; SILVA, N.M.; FERRAZ, C.A.M.; SCHMIDT, W.; NEVES, O.S.; RODRIGUES FILHO; F.S.O.; SABINO, N.P.; MARTINELLI, E.S.; LAZZARINI, J.F.; CORRÊA, F.A.; GROSSI, J.M.M.; CHIAVEGATO, E.J. Melhoramento do algodoeiro no Estado de São Paulo: obtenção da 'IAC RM3', 'IAC RM4', 'IAC 16' e 'IAC 17'. Bragantia, Campinas, v.43, n.2, p.405-423, 1984.

GRIDI-PAPP, I.L.; FUZATTO, M.G.; CIA, E.; SABINO, N. P.; KONDO, J. I.; GONDIM, R.M.A.; CAVALERI, P.A.; SILVA, N.M.; CARVALHO, L.H.; RODRIGUES FILHO, F.S.O.; WIESEL, J.B.C.; MARTINS, A.L M.; PETTINELLI JÚNIOR, A.; SORDI, G.De; SABINO, J.C.; PEREIRA, J.C.V.N.A.; CAMPANA, M.P.; BORTOLETTO, N.; GALLO, P.B. Ensaios regionais de variedades paulistas de algodoeiro VII- 1979/80- 1980/81. Campinas: Instituto Agronômico, 1993, 60p. (Boletim Científico, 27)

LAMAS, F.M.; FERRAZ, C.T. Resultados dos ensaios regionais paulistas, conduzidos em Mato Grosso do Sul, no ano agrícola de 1992/93. In: REUNIÃO NACIONAL DE ALGODÃO, 7., 1993, Cuiabá. Resumos.... Cuiabá: EMBRAPA/CNPA. 1993, p.35.

MEDEIROS, A.A. Resultado da produção de algodão nos ensaios de competição de linhagens e de cultivares do Rio Grande do Norte. In: REUNIÃO NACIONAL DO ALGODÃO, 8., 1995, Londrina. Resumos... Londrina: EMBRAPA/CNPA, 1995. p.34. 